\title{
Luminous IR Galaxies: Evolution and Molecular Gas
}

Yu Gao

Dept. of Astronomy, Univ. of Illinois, Urbana, IL 61801, USA

\section{Introduction}

Luminous infrared galaxies (LIRGs), defined by the criterion $L_{\mathrm{IR}} \gtrsim 2 \times 10^{11} L_{\odot}$ (for $H_{0}=75 \mathrm{~km} \mathrm{~s}^{-1} \mathrm{Mpc}^{-1}$ ), are the most powerful IR sources in the Universe, with most of their emission $(\sim 90 \%)$ in the far-IR. Most LIRGs are interact$\mathrm{ing} /$ merging galaxies with large amounts of molecular gas as revealed by $\mathrm{CO}$ surveys (Sanders et al. 1991; Solomon et al. 1996). However, whether starbursts or dust-enshrouded AGNs/QSOs dominate the IR luminosity is not resolved.

CO may not trace the active star-forming regions where gas density is more than one order of magnitude higher than the average. Dense molecular gas is better traced by high dipole-moment molecules like HCN and CS (e.g., NguyenQ-Rieu et al. 1992; Gao \& Solomon 1996). Therefore, it is essential to survey HCN emission in a large sample of LIRGs to better reveal the nature of LIRGs. We here study IR and molecular gas properties vs. galaxy-galaxy interactions in LIRGs over various merging phases to trace their evolution and explore some links among interactions, starbursts, and AGN phenomena.

\section{HCN-Far-Infrared Correlation}

We have surveyed $\sim 50$ 'normal' galaxies and LIRGs and found that LIRGs, especially ultraluminous galaxies, contain tremendous amount of dense molecular gas. The dense molecular gas content of even gas-rich 'normal' galaxies is much less than that of LIRGs of comparable molecular gas content. Fig. 1 shows a tight correlation between $L_{\mathrm{HCN}}$ and $L_{\mathrm{IR}}$ over three orders of magnitude. The correlation between $L_{\mathrm{CO}}$ and $L_{\mathrm{IR}}$, however, steepens with higher $L_{\mathrm{IR}}$. This suggests the star-formation rate depends on the amount of dense molecular gas traced by $\mathrm{HCN}$, not the total molecular content measured by CO (Gao 1996).

\section{Evolution of Luminous IR Galaxies Traced by CO}

We have found a correlation between the $\mathrm{CO}$ luminosity and the projected separation of merging nuclei in a sample of $\sim 50$ LIRG mergers (Fig. 2). This correlation suggests that the molecular mass of LIRG mergers is decreasing as the merger advances, and one interpretation is that the molecular gas content is being rapidly depleted due to the merger-induced starbursts while merging progresses to advanced stages (Gao 1996).

Since some LIRGs (especially those LIRGs in the advanced-merger stages) might be dust-enshrouded AGNs/QSOs and only a small molecular gas content or a lower detection rate has been found in AGNs/QSOs the decrease in LIRG's 


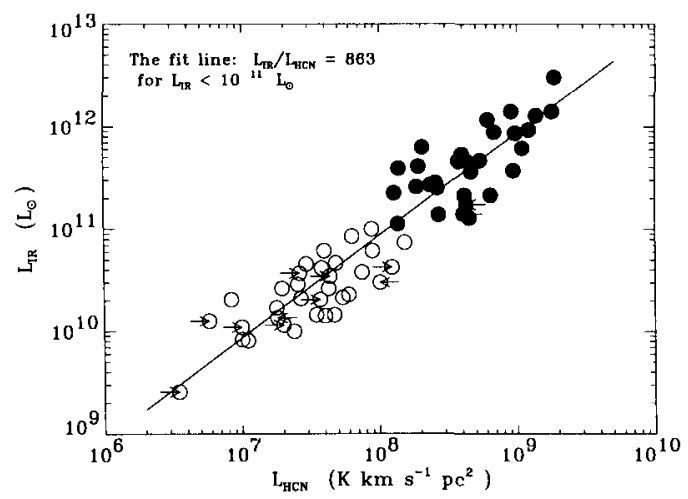

Figure 1. The correlation between HCN and IR luminosities.

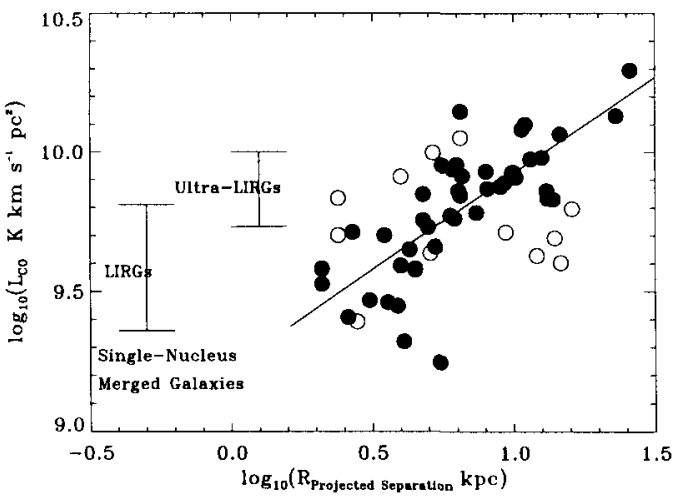

Figure 2. The correlation between $L_{\mathrm{CO}}$ and the projected separation.

molecular gas content as nuclei merge together might suggest an evolutionary connection between LIRGs and AGNs/QSOs. This correlation also provides an indirect evidence for the nuclear starburst-AGN connection.

We have started to image LIRGs in a merger sequence to explore the LIRGs over various merging phases using the newly expanded BIMA mm array. The first results on Arp 302 are presented by Lo, Gao, \& Gruendl (1996).

\section{References}

Gao, Y. 1996, Ph.D. thesis, SUNY at Stony Brook.

Gao, Y., \& Solomon, P. M. 1996, in preparation.

Lo, K.Y., Gao, Y. \& Gruendl, R. 1996, ApJ, submitted.

Nguyen-Q-Rieu, et al. 1992, ApJ, 399, 521.

Sanders, D. B., Scoville, N.Z., \& Soifer, B. T. 1991, ApJ, 370, 158.

Solomon, P. M., et al. 1996, ApJ, in press. 